3. Giachetti, A., Stanley, R. W., Zalubas, R. 1970, J. opt. Soc. Am., in press.

4. Goorvitch, D., Valero, F. P. J., Clúa, A. L. 1969, J. opt. Soc. Am., 59, 971.

5. Hall, J. L. 1968, IEEE J. Quantum Electronics, QE4, 638.

6. Hanes, G. R., Baird, K. M. 1969, Metrologia, 5, 32.

7. Hanes, G. R., Dahistrom, C. E. 1969, Appl. Phys. Lett. 14, 362.

8. Kaufman, V., Humphreys, C. J. 1969, J. opt. Soc. Am., 59, in press.

9. Martin, W. C., Kaufman, V. 1970, J. Res. NBS, in press.

10. Mielenz, K. D., Nefflen, K. F., Rowley, W. R. C., Wilson, D. C., Engelhard, E. $\quad$ 1968, Appl. Opt., $7,289$.

11. Norlén, G. 1970, Metrologia, 6, 19.

12. Radziemski, Jr., L. J., Kaufman, V. 1969, J. opt. Soc. Am., 59, 424.

13. Ross, Jr., C. B. 1969, Ph.D. thesis, Purdue University.

14. Rowley, W. R. C., Ward, L. 1968, Metrologia, 4, 146.

15. Valero, F. P. J. $\quad 1968$, J. opt. Soc. Am., 58, 484, 1048.

\author{
B. EDLÉN \\ Chairman of the Committee
}

\title{
COMMITTEE 2: TRANSITION PROBABILITIES
}

A very great deal of work has been done during the last three years on the determination of atomic transition probabilities, and complete coverage here is impossible. Wiese and his staff at the National Bureau of Standards, Washington, have continued to collect all numerical results, and they can supply information as to what is available for any particular atom. They have published (1) a critical compilation of atomic transition probabilities for the atoms sodium to calcium (inclusive) in essentially the same way as an earlier compilation covered hydrogen to neon. At the time of writing a complete new bibliography is also in preparation (2), which will be published as NBS Special Publication 320 and which is complete up to June 1969. Review articles published include that of Layzer and Garstang (3) on theoretical allowed and forbidden transition probabilities, and the two volume conference report (4) covers beam foil spectroscopy (including lifetime measurements) in some detail. A fairly complete review of forbidden line transition probabilities was given by Garstang (5), where references to many original papers may be found. In the following we shall confine ourselves to mentioning a few areas of particular interest; detailed references can be traced through the bibliographies, reviews and abstracting journals.

On the experimental side much attention has been given to the direct measurement of atomic lifetimes, and many individual cases have been studied. Outstanding has been the work by the phase shift technique by Lawrence, Hesser, Cunningham, and others. Atoms studied include OI, NII, NeI, NeII, NeIII and AlI. The direct decay of atomic states has been observed in other cases after pulsed electron excitation, after direct optical excitation and after beam-foil excitation. A major problem in these methods is cascading from higher states, and this limits the applicability of the methods, but there are many important states which have been, and could be, studied. As examples of what has been done we quote the work of Lawrence by pulsed electron excitation on ArI, ArII, ArII, ArIV, SII, ClII and XeI, of Wares and his group on various stages of ionization of oxygen by beam-foil spectroscopy, and of Wolff and Davis on decay following optical excitation in CsI and NaI. The Hanle effect has been used very successfully by Gallagher for the resonance lines and infrared triplets in CaII, SrII and BaII, and by deZafra and Marshall for SnI and PbI. Absorption spectroscopy has been used in many investigations, for example the work of Huber and Tobey and of Grasdalen, Huber and Parkinson on FeI, FeII, CrI and CrII and the work of Parkes, Keyser and Kaufman on the resonance triplet of Or. Emission spectroscopy of stabilised arcs has been used by Bridges and Wiese and by Foster on the Sr and SII spectra. Particularly important are the papers by the Kiel group (6) on Fer and FeII, using a well stabilized argon arc with a small admixture of iron chloride. They derive solar abundances of 7.6 (on the scale with $\log H=12$ ) from their results, in agreement with coronal values.

On the theoretical side an important trend has been the inclusion of configuration interaction in 
calculations by Hartree-Fock techniques. Work by Fischer on the AlI isoelectronic sequence is of particular interest; she explained some unexpectedly low oscillator strengths in Sirl. For highly ionized atoms intermediate coupling calculations must be performed, and configuration interaction must often be included as well. Intermediate coupling calculations were reviewed in (3); among more recent papers we mention the work of Mendlowitz on TiII. Examples of configuration interaction calculations include papers by Froese on various stages of ionization of iron and silicon and of Weiss on $\mathrm{Cr}$ and $\mathrm{CI}$ and on AlI. A number of calculations of oscillator strengths including electron correlation effects have been made by Sinanoglu and his students.

Many problems remain to be studied. Perhaps the most important is in the area of the oscillator strengths of high-excitation-potential lines in atoms such as FeI. Independent measurements of oscillator strengths for some such lines by methods which do not involve a knowledge of Boltzmann factors are greatly desired to check on excitation potential effects in the existing measurements, and to place existing measurements on a more reliable absolute scale.

Recent work on forbidden lines was reviewed in (5). Confidence in the reliability of the theoretical results has been increased to some extent by Thackeray's comparisons of [Fem] and [NiII] intensities in $\eta$ Carinae with theoretical intensities. Laboratory experiments by Hults on [PbI] and [PbII] lines in which there is a mixture of magnetic dipole and electric quadrupole radiation tend to confirm the theoretical ratios of these two types of radiation. Measurements of some ratios of lines in [OI] and in [SI] by LeBlanc, Oldenberg and Carleton, and by McConkey, Burns, Moran, Kernahan and Emeleus are in excellent agreement with the theory. Garstang has calculated the transition probabilities for a number of magnetic quadrupole transitions, and showed that one line in each of Ferx, FexvII and Fexxv may be a significant contributor to the de-excitation process in these ions in the solar corona. The $2{ }^{3} S_{1}-1{ }^{1} S_{0}$ line in the helium isoelectronic sequence has been shown by Griem to be due to magnetic dipole radiation made possible in a relativistic approximation.

\section{REFERENCES}

1. Wiese, W. L., Smith, M. W., Miles, B. M. 1969, Nat. Stand. Ref. Data System - NBS 22. Atomic transition probabilities, Vol. II.

2. Miles, B. M., Wiese, W. L. 1970 , Nat. Bur. Stand. Special Pub. 320.

3. Layzer, D., Garstang, R. H. 1968, A. Rev. Astr. Astrophys., 6, 449.

4. Bashkin, S. 1968, Beam-foil Spectroscopy, 2 vols., Gordon and Breach, New York.

5. Garstang, R. H. 1969, Mém. Soc. r. Sci. Liège, 17, 35-44.

6. Baschek, B., Garz, T., Holweger, H., Richter, J. $\quad 1969$, Astr. Astrophys. 2, 274-279 and 446-450; 1970, Astr. Astrophys. 4, 229-233.

R. H. GARSTANG

Chairman of the Committee

COMMITTEE 3: COLLISION CROSS SECTIONS AND LINE BROADENING

\section{LINE BROADENING}

Two review articles have been published $(1,2)$.

1.1. Collision broadening by neutral atoms. The continued study of widths and shifts produced by neutral atom impacts at low densities has shown that the Van der Waals interaction $\left(-\mathrm{C}_{6} r^{-6}\right)$ as used, for example, in (3), is an inadequate representation of the interatomic potential. The classical theory for a Leonard-Jones potential $\left(\mathrm{C}_{12} r^{-12}-C_{6} r^{-6}\right)$ has been developed (4) and subjected to experimental test (5). Further improvements result from introducing a term $\mathrm{C}_{8} r^{-8}$ in the potential (6) and from allowance for quasi-static effects (7). Applications to broadening by $\mathrm{H}$-atom impact are discussed in (8).

Progress has been made in the study of resonance broadening (collisions between atoms of the 